\title{
The PIV study of air flow past the counter- swirler 53983
}

\author{
Daniel Duda ${ }^{1, *}$ \\ ${ }^{1}$ Department of Power System Engineering, Faculty of Mechanical Engineering, University of West \\ Bohemia in Pilsen, Univerzitní 22, 30614 Plzeň, Czech Republic
}

\begin{abstract}
PIV (particle image velocimetry) measurement of the air flow past a counter-swirler 53983 (anticlockwise swirler surrounded by clockwise swirler) is performed. The measurement is focused to an area at the boundary between the inner swirling jet and the outer one rotating oppositely. The Reynolds number Re based on the inner swirler diameter ranged form $1.2 \cdot 10^{3}$ to $2.1 \cdot 10^{4}$. By using band pass filtering the shear layer and vortices in the contact region between counter-swirling jets is highlighted. The shear layer between these regions shortens and decays into vortices as Reynolds number increases.
\end{abstract}

\section{Introduction}

Free jet into a liquid usually produces turbulence due to the Kelvin-Helmholtz instability in the free sheer layer between the moving jet and the surroundings. In mixing studies, the jet can be swirled by using a fixed propeller in the nozzle, which widens the jet profile and speeds up the turbulence evolution [1]. This is in the literature refereed as a swirling jet [2]. If one would like to avoid net angular momentum in the downstream [3], then the counterswirler consists of a inner-ring and a oppositely oriented outer-ring of blades resulting into a pair of concentric jets rotating oppositely.

From the physical point of view, this kind of flow is interesting because it combines the Kelvin-Helmholtz instability with rotation, or, from the second side, it can be seen as a Taylor-Couette instability between "fluidic walls".

The aim of this study is to perform a preliminary preview on this kind of flow generated by a mass-produced component number 53983 manufactured by the company "Lego". The geometry of the part 53983 seems to be far from ideal, Figure 1, and its planed improvement will be based on the here published results.

\section{Experimental setup}

A standard method of Particle Image Velocimetry (PIV) has been used; the method is based on suspending small particles (in this case of oil) into the studied fluid (in this case air) and believing that these particles follow the motion of the fluid [4]. These particles are

\footnotetext{
*Corresponding author: dudad@kke.zcu.cz
} 
illuminated by a laser sheet and observed from direction perpendicular to this sheet. PIV does not allow direct determination of velocity of an individual particle, instead it provides a statistical estimate of the Eulerian velocity field in an arbitrary interrogation areas (IA) with a small number $(\sim 10)$ of particles. The velocity in one IA is calculated as the shift of a cross-correlation peak between two consecutive photographs of the particles illuminated by a laser. The time between these frames is set according to the velocity and, in this case, it ranges from 20 to $160 \mu \mathrm{s}$. As the computational algorithm, the function "Adaptive PIV" performed by the "Dantec Dynamic studio" has been used.

The flow is generated by the wind tunnel borrowed by the company "WT-tech", and the obstacle (counter-swirler 53983) is located at the beginning of the measurement volume of square cross-section of $125 \mathrm{~mm}$ side. The illuminated plane is oriented along the flow and crosses the center of the obstacle.
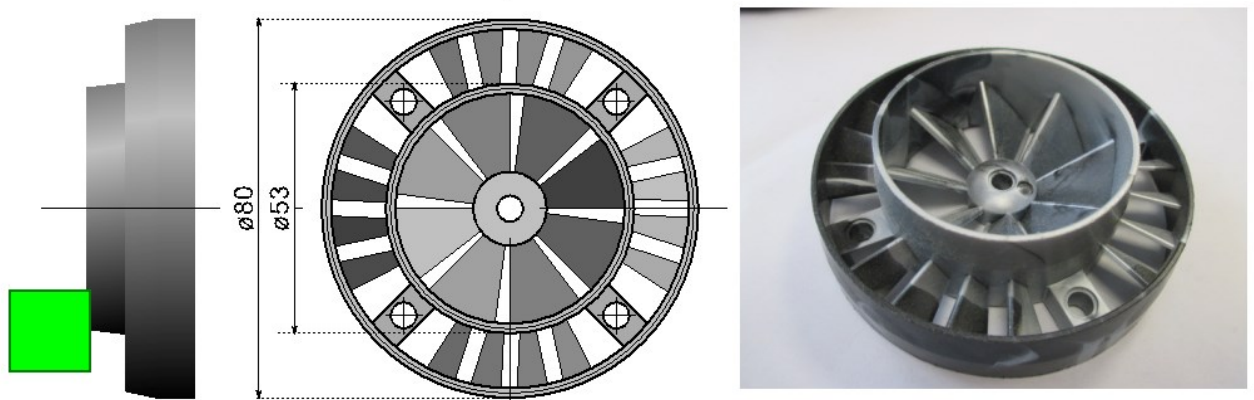

Fig. 1. Sketch of the counter-swirler 53983, units in $\mathrm{mm}$. The green rectangle of size $17.4 \times 17.4 \mathrm{~mm}$ highlights the smaller studied plane. Right: photograph of the device.

\section{Observations}

\subsection{Large-scale overview}

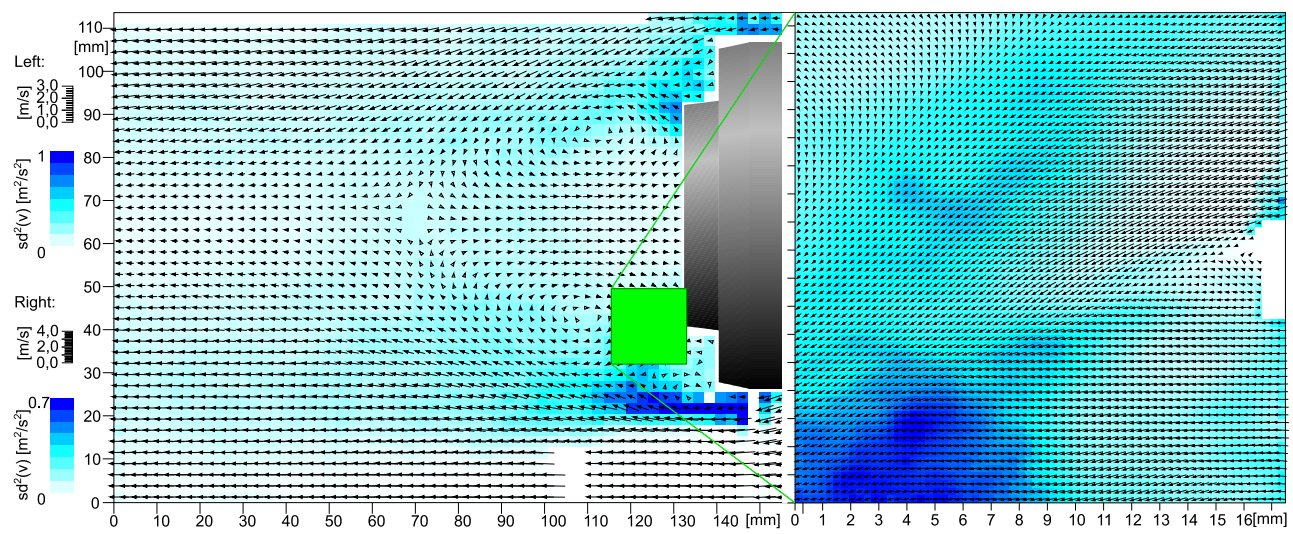

Fig. 2. Mean flow field past the obstacle (sketched in the figure). The color corresponds to the fluctuating energy content. Left: the large-scale view, right: the region of interest drawn as a green rectangle $17.4 \times 17.4 \mathrm{~mm}$.

First lets look at the large-scale structure of the flow past the obstacle, Figure 2-left. Scale of this measurement is $2.58 \mathrm{~mm} / \mathrm{IA}$ showing the overview of the wake, but at this scale it is impossible to deal with the structures in fluctuating field. We can see a pair of big vortices (would be a big vortex ring in 3D) of recirculating fluid behind the obstacle. At this 
scale the flow is dominated by the regime "flow past a disk" and the counter-swirling character is hidden at larger distances from the swirler. In shorter distance, there can be observed the centrifugal flow widening [5]. Therefore, a smaller region of interest has been selected close to the obstacle just at the boundary between the clockwise and counterclockwise swirling regions, see figure 2-right. The fact the swirler is situated like a bluff obstacle is not a problem there. The corresponding scale is $273 \mu \mathrm{m} / \mathrm{IA}$.

\subsection{Detailed look}

Figure 3 shows the dependence of Intensity of turbulence $I_{\mathrm{T}}$ usually defined as

$$
I_{T}=\frac{\sqrt{\left\langle\left(\mathbf{v}-\langle\mathbf{v}\rangle_{T}\right)^{2}\right\rangle_{T}}}{\left|\langle\mathbf{v}\rangle_{T}\right|}=\sqrt{\frac{E_{\mathrm{RR}}}{E_{\mathrm{SP}}}}
$$

on the Reynolds number $R e$ based on the inner swirler diameter $D_{\text {in }}=53 \mathrm{~mm}$. In equation (1) $\langle\cdot\rangle_{\mathrm{T}}$ means the averaging in respect to time, which is in this case, due to quite small camera frame-rate, averaging over independent realizations. $E_{\mathrm{RR}}$ means the mean energy content of the fluctuating field obtained by the so called Reynolds decomposition: $\mathbf{v}^{\prime}(t)=$ $\mathbf{v}(t)-\langle\mathbf{v}\rangle_{\mathrm{T}}$, and $E_{\mathrm{SP}}$ is the energy content of the mean field, of-course, they must fulfill that $E=E_{\mathrm{SP}}+E_{\mathrm{RR}}$, where $E$ is the total kinetic energy content ${ }^{\dagger}$.
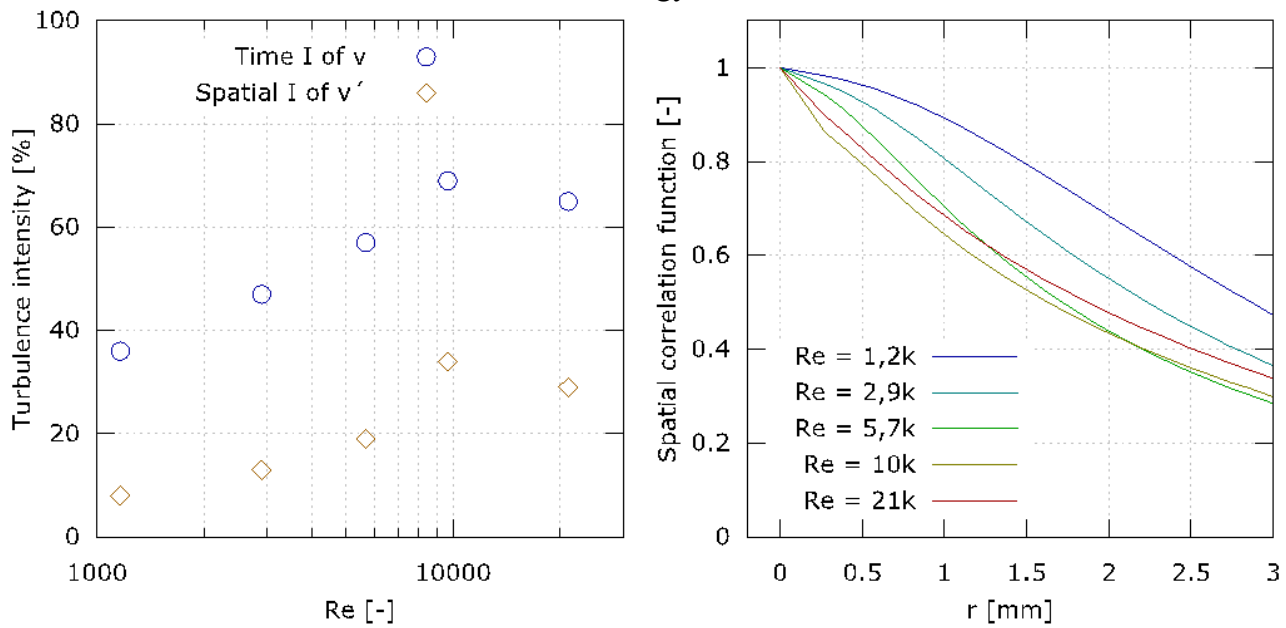

Fig. 3. Left: blue circles: the dependence of turbulence intensity, equation (1), on the Reynolds number $R e$ based on the measured velocity and the inner swirler diameter $D_{\text {in }}=53 \mathrm{~mm}$. Brown diamonds: spatial turbulence intensity in respect to 1 IA Gaussian filtering of the fluctuating velocity field, equation 3 i.e. a measure of loses by smoothing. Right: the spatial correlation function $R(r)$, equation (2), for different $R e$. Note the lack of inflexion point for higher $R e$. The value of $R(r)$ is normalized by its value at $r=0$, which corresponds to the energy of fluctuating component $E_{\mathrm{RR}}$.

In Figure 3-left one can see the drop of $I_{T}$ at the highest $R e$ point. This is probably caused by the insufficient spatial resolution, which misses lot of energy in fluctuating field because this energy is in length-scales smaller than is the current resolution. Note that PIV provides velocity in one IA, which is already averaged over this IA. This problem is alternatively illustrated in figure 3-right, where the spatial correlation function $R(|\mathbf{r}|)$ is plotted.

${ }^{\dagger}$ Lets explain the strange unit $\left[\mathrm{J} \mathrm{m} \mathrm{m}^{2} / \mathrm{kg}\right]$ used in this paper: $E=1 / 2 \rho \int \mathbf{v}^{2} \mathrm{~d} V$, where $\mathrm{d} V=$ $\mathrm{d} x \cdot \mathrm{d} y \cdot \mathrm{d} z$ and only $\mathbf{v}, \mathrm{d} x$ and $\mathrm{d} y$ are measured, $\rho \approx 1.2 \mathrm{~kg} / \mathrm{m}^{3}$ is the density of air and $\mathrm{d} z \approx$ $1 \mathrm{~mm}$ is the thickness of the laser sheet, but these quantities are not measured, therefore we publish only safe numbers: $E=E_{\text {real }} / \rho / \mathrm{d} z$. 


$$
R(\mathbf{r})=\left\langle\mathbf{v}^{\prime}(\mathbf{x}) \cdot \mathbf{v}^{\prime}(\mathbf{x}+\mathbf{r})\right\rangle_{S},
$$

where the subscript $S$ denotes spatial averaging and $\mathbf{v}$ ' is the already Reynolds-decomposed velocity field. We can see that for larger velocities, the curve lacks inflexion point -- it is convex since the origin saying that parabolic region is smaller than one IA.

\subsection{Looking for middle-scale structures}

Figure 4-left shows the Reynolds decomposed velocity field convoluted with Gaussian with $\sigma=1 \mathrm{IA}=0.27 \mathrm{~mm}$. The convolution with Gaussian averages out structures smaller than $\sigma$ in order to kill the noise, which is uncorrelated, but on the other hand we loose physical information at this length-scale, which anyway is indistinguishable from the noise. The measure of loses caused by this procedure in sense of turbulence intensity is shown in figure 3-left as brown diamonds and calculated as

$$
I_{S}=\frac{\sqrt{\left\langle\left(\mathbf{v}^{\prime}-\mathbf{v}^{\prime} * G_{\sigma}\right)^{2}\right\rangle_{S}}}{\sqrt{\left\langle\mathbf{v}^{\prime} * G_{\sigma}^{2}\right\rangle_{S}}}=\sqrt{\frac{E_{1-\mathrm{G} 1}}{E_{\mathrm{G} 1}}} .
$$

Highlighting the middle-scale structures can be achieved via the convolution with a band-pass filter [6] referred as Agrawal decomposition, which removes structures larger than its upper width, see figure 4-right. The filter is just difference of two Gaussians with widths $\sigma_{\mathrm{L}}$ and $\sigma_{\mathrm{H}}$ :

$$
P(\mathbf{x})=G\left[\sigma_{L}\right](|\mathbf{x}|)-G\left[\sigma_{H}\right](|\mathbf{x}|)=\frac{1}{\sqrt{2 \pi}}\left(\frac{1}{\sigma_{L}} \mathrm{e}^{\mathbf{x}^{2} / 2 \sigma_{L}^{2}}-\frac{1}{\sigma_{H}} \mathrm{e}^{\mathbf{x}^{2} / 2 \sigma_{H}^{2}}\right) .
$$
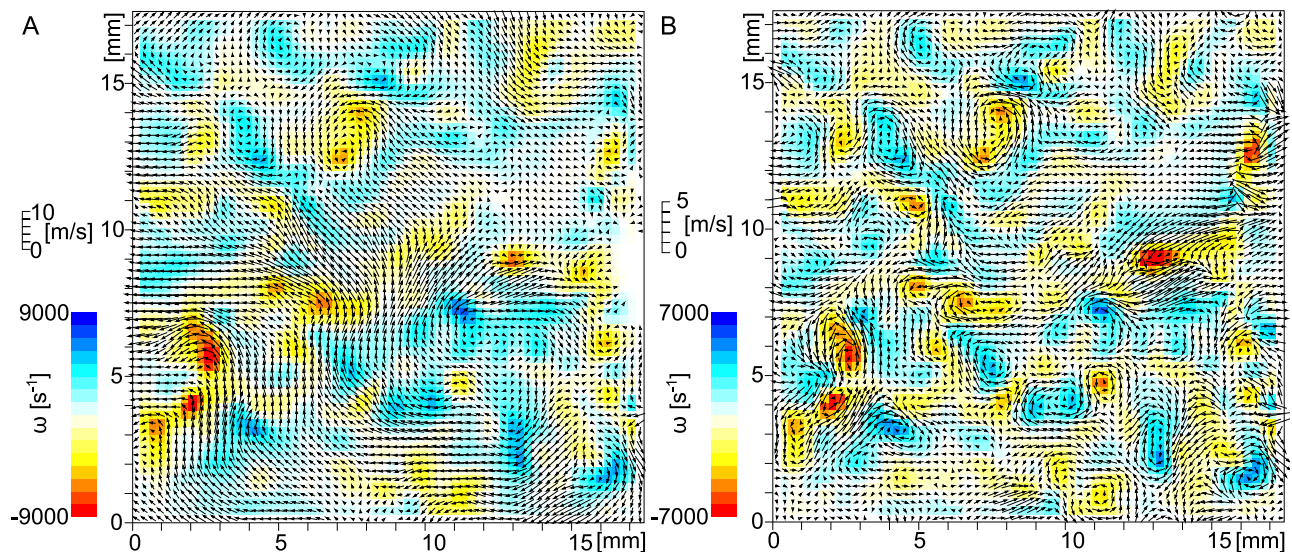

Fig. 4. Left (A): the Reynolds decomposed velocity field in the smaller studied area (green rectangle in figure 1) convoluted with Gaussian with $\sigma=0.27 \mathrm{~mm}$. Right (B): The Agrawal decomposition, i.e. original velocity field convoluted with band pass filter with upper width $\sigma_{\mathrm{H}}=1.1 \mathrm{~mm}$. Both pictures show the same particular realization of the flow with $R e=10^{4}$. Color in both cases corresponds to classical vorticity.

The particular similarity of both pictures suggests that the fluctuating component consist of such sized structures, but not only of them, as the energy content of the particular case in figure 4-left is $E_{\mathrm{RR}}=0.9 \mathrm{~mJ} \cdot \mathrm{m}^{2} / \mathrm{kg}$, while for the band-pass filtered data it is $E_{\text {Pas }}=0.13$ $\mathrm{mJ} \cdot \mathrm{m}^{2} / \mathrm{kg}$, for comparison, the energy of the mean field is $E_{\mathrm{SP}}=2.2 \mathrm{~mJ} \cdot \mathrm{m}^{2} / \mathrm{kg}$. The value of the upper width of the band filter, which is in the presented figure $\sigma_{\mathrm{H}}=1.1 \mathrm{~mm}$, is justified by the Figure 3-right showing that the spatial correlation function crosses the value of $1 / 2^{1 / 2} \sim$ $71 \%$ approximately at such distance. While the Agrawal decomposition shows nicely small vortices, the Reynolds decomposition in addition shows larger masses moving coherently, 
which is naturally responsible for some of energy difference between Reynolds and Agrawal decomposed fields.
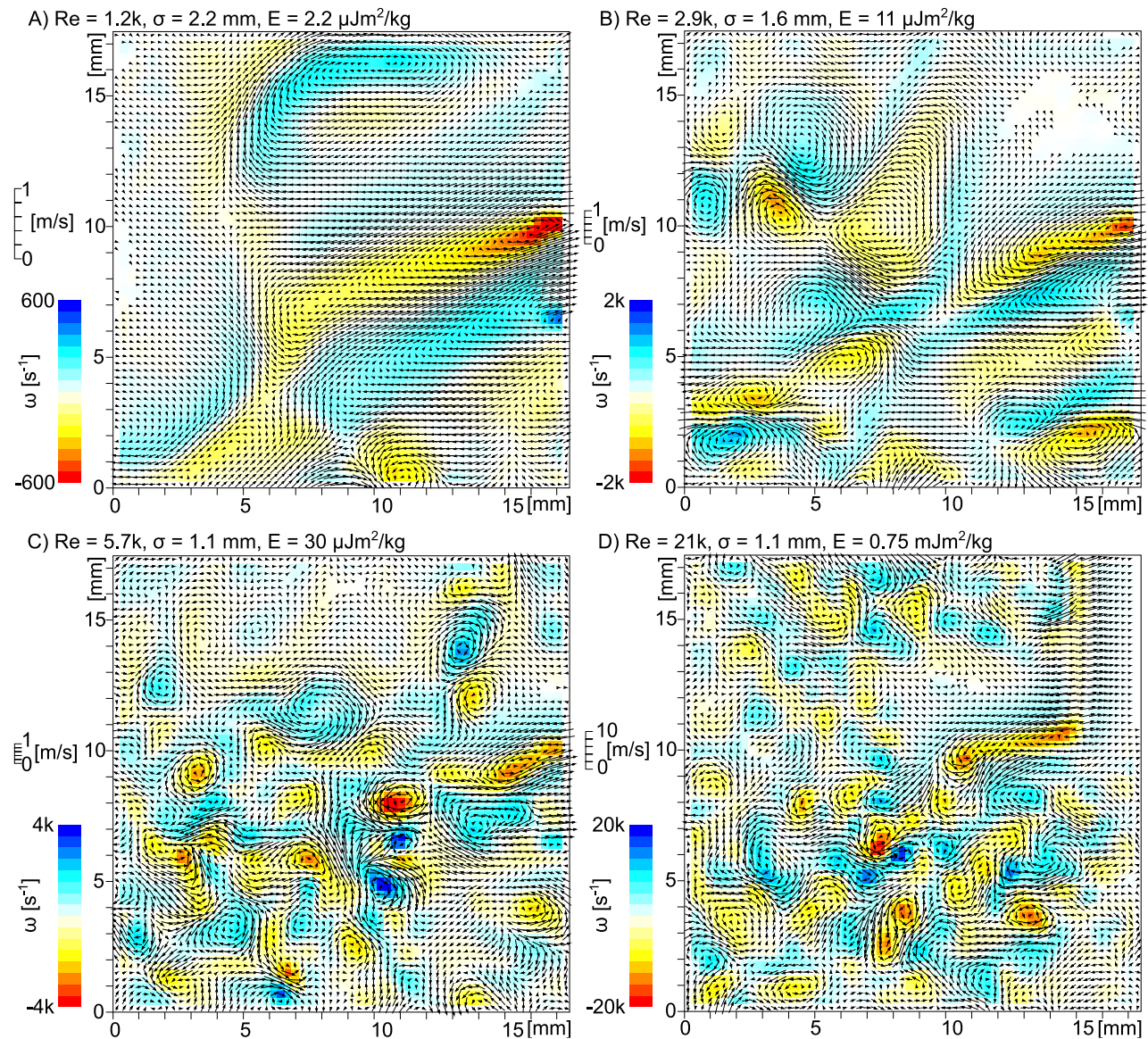

Fig. 5. The band-pass filtered velocity field for different Reynolds numbers. $\sigma$ is the band upper width chosen according to the spatial correlation function.

Figure 5 shows band-filtered velocity field of other cases. For smaller $R e$ the larger $\sigma_{\mathrm{H}}$ has been chosen according to the spatial correlation function, Figure 3-right. Lets note one of the disadvantages of the Agrawal decomposition -- it highlights the areas of large velocity gradient as if it would be shear layers, especially if these are larger than the band upper width. On the other hand, at higher Re and more intensive turbulence with many overlapping vortices of different sizes, the Agrawal decomposition makes visible also smaller vortices carried by larger ones [7], because it subtracts the correct local advection velocity and it is not needed to estimate it manually [8]. This makes this method better for the vortex identification, than is the standard Reynolds decomposition.

In Figure 5 we can see shortening of the shear layer between clock- and anticlockwise swirling region with increasing $R e$. At the smallest one, this layer is about $1 \mathrm{~cm}$ long, at Re $=2.9 \mathrm{k}$ it is about $7 \mathrm{~mm}$ and at $R e=5.7 \mathrm{k}$ it shortens down to $3 \mathrm{~mm}$, while it is difficultly recognizable at larger $R e$. Behind this region, the shear layer decays into vortex tangle. 


\section{Conclusion}

The flow field past a double concentric counter-swirler of type 53983 has been visualized by using a standard Particle Image Velocimetry (PIV) technique. The measurement was focused to a small area behind the swirler at the boundary between the inner swirling jet rotating anticlockwise and the outer swirling jet rotating clockwise. The Reynolds number $R e$ based on the inner swirler diameter ranged from $1.2 \cdot 10^{3}$ to $2.1 \cdot 10^{4}$. By using band pass filtering suggested by Agrawal [6] the shear layer and vortices in the contact region between counter-swirling jets has been highlighted. The shear layer between these regions shortens and decays into vortices as Reynolds number increases. The spatial correlation function analysis states, that for the largest $R e$ the used spatial resolution is inadequate and has to be increased for future experiments.

This work was supported by the project SGS-2016-045 (Increase of efficiency, reliability and a lifespan of power machines and devices 4). The author thanks Martin Duda working at WAT in Warsaw for providing the Lego part number 53983 used in this study.

\section{References}

1. M.R. Ruith, E. Meiburg, Journal of Turbulence 3, 37 (2002)

2. F. Cozzi, A. Coghe, R. Sharma, Experimental Thermal and Fluid Science 94, 281 (2018)

3. H. Werschnik, M. Schneider, J. Herrmann, D. Ivanov, H.P. Schiffer, C. Lyko, International Journal of Turbomachinery, Propulsion and Power 2, 12 (2017)

4. V. Uruba, O. Hladík, P. Jonáš, Journal of Physics: Conference Series 318 (2011)

5. C.E. Cala, E.C. Fernandes, M.V. Heitor, S.I. Shtork, Experiments in Fluids 40, 267 (2006)

6. A. Agrawal, A. Prasad, Experiments in Fluids 33, 565 (2002)

7. A. Agrawal, Experiments in Fluids 39, 836 (2005)

8. R.J. Adrian, K.T. Christensen, Z.C. Liu, Experiments in Fluids 29, 275 (2000) 